\title{
Video Article \\ Assessment of GFP Expression and Viability Using the Tali Image-Based Cytometer
}

\author{
Krissy Remple ${ }^{1}$, Laurel Stone ${ }^{2}$ \\ ${ }^{1}$ Department of Molecular and Cell Biology, Life Technologies \\ ${ }^{2}$ Life Technologies \\ Correspondence to: Krissy Remple at Krissy.Remple@lifetech.com
}

URL: https://www.jove.com/video/3659

DOI: doi:10.3791/3659

Keywords: Cell Biology, Issue 57, cytometry, imaging, image-based, cell viability, apoptosis, cell counting, expression assay, Dead-Cell Red, propidium iodide, PI, GFP expression, RFP expression, cell analysis, fluorescence protein expression

Date Published: $11 / 17 / 2011$

Citation: Remple, K., Stone, L. Assessment of GFP Expression and Viability Using the Tali Image-Based Cytometer. J. Vis. Exp. (57), e3659, doi:10.3791/3659 (2011).

\section{Abstract}

Single-cell and population information are commonly obtained either by flow cytometry or fluorescence microscopy. However, these two methods provide different information. Flow cytometry gives quantitative multi-parametric information about physical characteristics and staining or expression, but doesn't allow for visualization. Stand-alone fluorescence microscopy provides visual data, but doesn't allow for straightforward quantitative measurements ${ }^{1}$.

Image-based cytometry bridges the gap between these two methods, enabling the quick visualization and simultaneous quantitative analysis of thousands of cells in heterogeneous populations ${ }^{2}$. Here, we present a method for performing cell viability and green fluorescent protein (GFP) expression assays using the Tali Image-Based Cytometer ${ }^{3}$. The Tali instrument is a 3-channel (bright field, green fluorescence, red fluorescence) benchtop assay platform that offers several advantages over flow cytometry and fluorescence microscopy. The Tali cytometer is less expensive, takes up less bench space, requires less maintenance, and the work flow has been simplified so that the operation and analysis is much simpler and quicker. The Tali cytometer is capable of performing a range of suspension cell-based assays, including GFP and red fluorescent protein (RFP) expression, apoptosis ${ }^{4-6}$ and cell viability analysis with propidium iodide $(\mathrm{PI})^{7-11}$.

Here, we demonstrate the use of the Tali instrument in performing a cell viability assay in cells expressing GFP. GFP-transduced cells are stained using the Tali Viability Kit - Dead Cell Red. The cells are then pipetted into a Tali Cellular Analysis Slide and loaded into the cytometer. Bright field, red fluorescence and green fluorescence images are captured and analyzed using assay specific algorithms. Histograms are then generated to display cell size, PI fluorescence intensity, and GFP fluorescence intensity. These parameters can then be thresholded to home in on a specific cell population.

A side-by side comparison of the Tali Image-Based Cytometer and traditional flow cytometry demonstrates that the two methods provide comparable data regarding cell viability and protein expression. However, the Tali instrument provides additional visual information about the cell population that cannot be obtained using a flow cytometer.

\section{Video Link}

The video component of this article can be found at https://www.jove.com/video/3659/

\section{Protocol}

\section{Staining Cells with the Tali Viability Kit - Dead Cell Red Reagent}

1. Begin this procedure with U2OS cells (American Type Culture Collection) that have been transduced using CellLight Nucleus-GFP *BacMam $2.0^{*}$. (Note: Cells can also be spun down and resuspended in medium or PBS).

2. To stain dead cells with PI, transfer $100 \mu \mathrm{L}$ of the cells to a new microcentrifuge tube. Note that a concentration of $1 \times 10^{5}$ to $1 \times 10^{7}$ cells/mL is recommended for this assay, though the concentration does not have to be exact.

3. Next, to stain the dead cells, add $1 \mu \mathrm{L}$ of the PI-based Tali Dead Cell Red reagent. Vortex briefly to mix. Incubate the Tali Dead Cell Red reagent and cell mixture at room temperature in the dark for 1-5 minutes.

4. Following the incubation, immediately proceed to analysis of the sample on the Tali Image-Based Cytometer.

\section{Performing a Cell Viability Assay Using a Tali Image-Based Cytometer}

1. The Tali cytometer uses the disposable plastic Tali Cellular Analysis Slides that specifically fit into the cytometer and can hold two $25 \mu \mathrm{L}$ samples in separate enclosed chambers (A and B). When handling the slide, be sure to always hold it by the sides. 
2. To load the slide, pipette $25 \mu \mathrm{L}$ of sample slowly into the half moon-shaped sample loading area, taking care to avoid forming bubbles or back splatter. Do not over or under fill.

3. Here, control cells (unstained, non-transduced) are loaded in chamber A and the stained GFP-expressing cells are loaded in chamber B. The control sample will help determine the level of autofluorescence when analyzing the data.

4. To perform a viability assay, touch GFP/RFP on the home screen of the cytometer. The assay options will then appear on the right. Select GFP + Viability.

5. Next, to name the sample series, press Name Now. Then, using the touch screen, type the name of the sample series, and press Save. (Note: Select Name Later to automatically assign a name for each sample series by date and time.)

6. After naming the sample, the Tali cytometer will automatically advance to the Measure screen. Press the Background tab on the bottom right half of the Measure screen.

7. Next, with the control sample (A) facing away from the cytometer, insert the slide in the loading port until it stops. Do not apply force.

8. On the background screen, touch Press to Insert New Unstained Cell Control. The slide will automatically be pulled into the cytometer and a live image of the cells will be displayed on the screen.

9. Using the focus knob, bring the cells into the proper plane of view. The cells should be uniformly dark with a bright halo around each cell (Figure 1, Left).

10. Cells may be undercounted when the transition between the background and the edges of the cells is fuzzy, so that cell boundaries are not well defined (Figure 1, Center). Cells may be overcounted when they have bright centers and dark perimeters (Figure 1, Right).

11. To better view the cell population while focusing, slide the red Zoom indicator button to $4 \mathrm{X}$ or $16 \mathrm{X}$

12. Once the cells have been brought into focus, touch Press to Run Unstained Cell Control to measure the background fluorescence.

13. The cytometer will automatically capture and analyze the images of the sample. It takes about 1 minute to capture and analyze the images in the default (9 image) mode.

14. Thumbnails of each field of view are displayed as they are captured and the progress bar provides an ongoing update. After image capture is complete, the cytometer automatically ejects the slide and provides the data from the analysis of the captured images.

15. The stored data will be displayed in the drop down menu of the Background tab on the cytometer. The background control sample will be assigned the same name as that given to the experiment and will be imported in the drop down menu. Note: If a background control is not run, the cytometer automatically assigns a fluorescence threshold. This can be manually changed after performing the assay.

16. To run the PI-stained transduced sample, remove the slide from the cytometer, flip it around and reinsert it with the transduced sample facing away from the instrument.

17. Press the Sample tab, and then touch Press to Insert New Sample. When the image appears on the screen, use the image adjustment knob to bring the cells into focus as before. Then touch Press to Run Sample.

18. After image capture is complete, the data from the analysis appears under the Sample tab and the cytometer automatically ejects the slide.

19. Appropriately dispose of the Tali Cellular Analysis Slide as biohazardous waste. Tali Cellular Analysis Slides cannot be reused.

\section{Setting Thresholds and Gates}

1. Once the sample has run, thresholds can now be applied to the sample to home in on a specific cell population. Here, we will adjust the thresholds to determine the percentage of live and dead cells in GFP-transduced cells.

2. Under the Sample tab, the number and percentage of cells expressing GFP, live and dead cells expressing GFP, total viability, average cell size, number of cells counted, and cell concentration are displayed.

3. In addition, histogram plots displaying cell size, green fluorescence, and red fluorescence (PI) are displayed at the bottom of the screen

4. To set the cell size gate, touch the Cell Size thumbnail to open the cell size histogram. While cultured cells rarely have debris, other samples may contain debris that is larger or smaller than the cells.

5. To exclude this debris, slide the blue buttons on the slider bar to exclude both large and small events. Then, touch Apply to re-analyze the data and update the results.

6. Next, to add the GFP fluorescence to the analysis, touch the GFP histogram thumbnail to open it. Slide the blue bar to set the threshold just to the right of the dimmest peak, using the control sample as a guide.

7. This allows the analysis to include the GFP positive cells, but exclude autofluorescent cells. Autofluorescence is frequently observed when biological molecules within cells are excited.

8. Touch Apply to confirm and return to the previous screen. The data displayed under the Sample tab should now reflect the gates and thresholds set for both fluorescence channels.

9. Next, to separate the PI stained cells from autofluorescence, press the PI histogram thumbnail to open the PI histogram.

10. Again, the control sample peak will be displayed as a gray peak on the histogram. The red peak is the sample fluorescence. The background fluorescence is displayed as a peak closest to the 0 RFU value on this cytometer.

11. To eliminate the background fluorescence in the PI channel, move the blue button on the slider bar to adjust the threshold to exclude the peak representing background fluorescence, using the gray peak from the control sample as a reference. Touch Apply to confirm and return to the previous screen.

12. Next, to visually confirm that each cell is properly assigned, select a captured field of view for review by pressing the thumbnail of the image in the Zoom tab, then pressing the Layers tab.

13. Next, press the GFP or PI button, to display the image captured through that particular channel. Press the same button again to remove the layer from the display; or, to superimpose layers, touch the Multiple Layer button.

14. To identify how the cells are counted through a particular channel, touch Circles. The cells that were counted through only the bright-field channel, which do not express fluorescence will be circled in blue. This indicates that that particular cell is live (i.e. PI negative).

15. Cells expressing GFP will be seen in the GFP channel, and will be circled in green. Dead cells stained with Dead Cell Red will be seen in the PI channel, and will be circled in red.

16. Cells counted in both the red and green channel will be circled in yellow, this indicates that the cell expresses GFP, but is also stained with PI and therefore is dead.

17. Occasionally, black circles will appear on the screen, these are objects that have been identified, but have been excluded from the analysis based on cell size. 
18. Continue to fine tune the threshold on the fluorescence histogram using the steps just described until each cell that is expressing fluorescence is circled with the appropriate color.

19. All analysis parameters are automatically saved under the data tab. The Tali cytometer can store data files from approximately 500 sample runs.

20. Each file stored in the data tab is available for reanalysis. By selecting the file from the Data tab it will be opened and all histograms and layers become active.

21. To archive data and generate reports, the data stored in the cytometer may be transferred to a computer using a USB drive.

\section{Representative Results:}

The Tali Image-Based Cytometer was used to measure the fluorescent protein expression level in cells that were transduced with a nucleartargeted GFP BacMam 2.0 expression construct. Four representative cell types were transduced (U2OS, 293MSR, HEKn, and CHO-S). For these cell lines, the number of cells that were expressing GFP was reported by the Tali cytometer and compared with data from the same samples analyzed on a flow cytometer. Additionally, cells were stained with Dead Cell Red reagent using the Tali Viability Kit to identify the dead cell population both on the Tali cytometer and on a flow cytometer.

The graphical representations in Figure 2 show the percent of the total population for each cell type that were expressing GFP. These data demonstrate that the Tali cytometer produces data comparable to data generated by a flow cytometer.
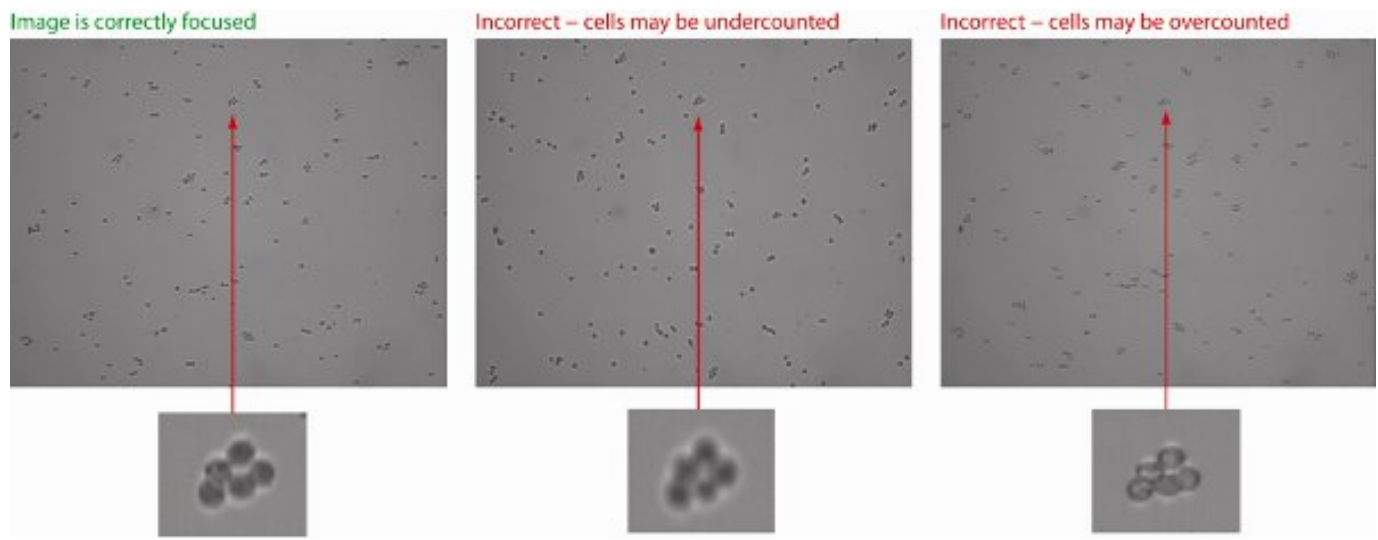

Figure 1. Cells should be properly focused prior to analysis. Tali Cellular Analysis slides with cell concentrations appropriate for analysis $\left(1 \times 10^{5}\right.$ to $1 \times 10^{7}$ cells are recommended). (LEFT) In-focus cells are uniformly dark throughout the cell, with a bright halo around each cell. (CENTER) Cells may be undercounted when the transition between the background and the edges of the cells are fuzzy and the cells have undefined boundaries (RIGHT) Cells maybe overcounted when they have bright centers and dark perimeters.

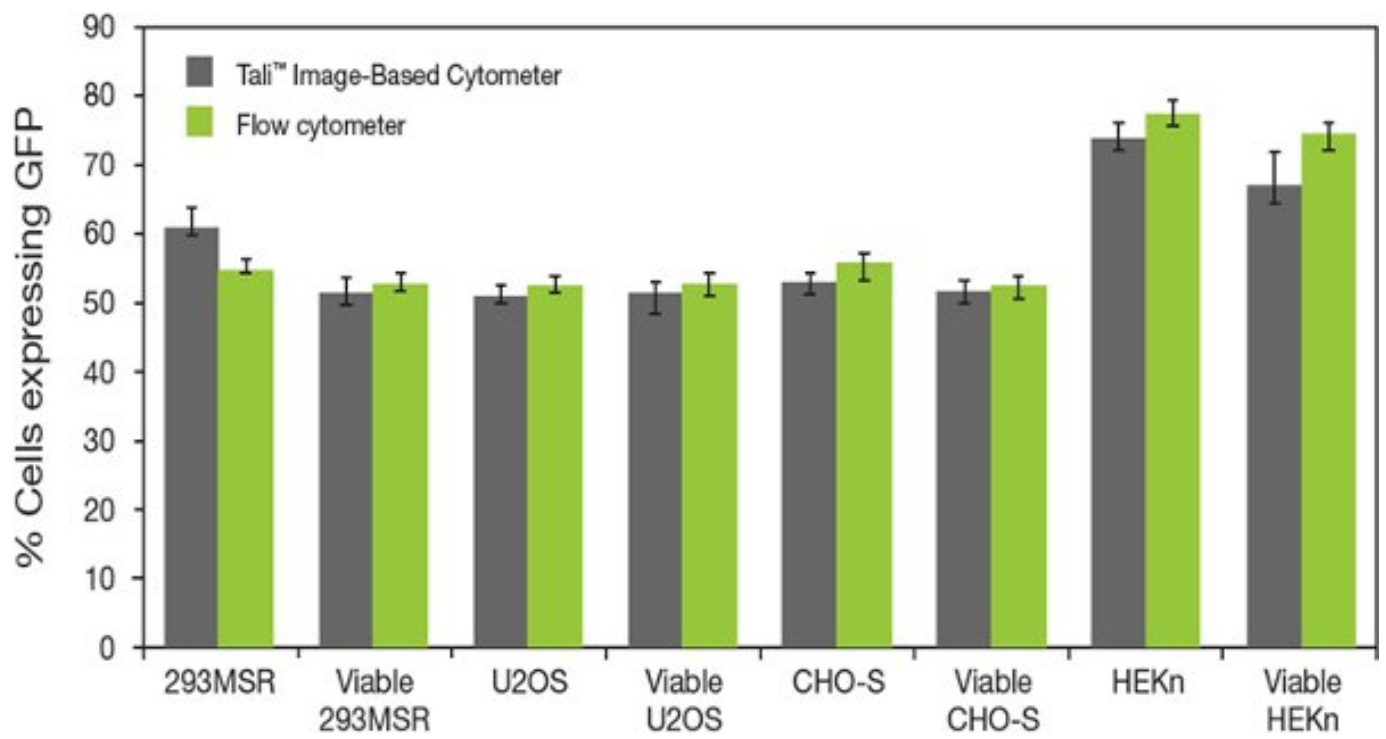

Figure 2. Fluorescent protein expression data from the Tali Image-Based Cytometer is comparable to that obtained using flow cytometry. A side-by-side comparison of calculated GFP-expression results in 4 different cell lines in viable cells. 


\section{Discussion}

We have just detailed a procedure for performing viability counts and assessing GFP expression using the Tali Image-Based Cytometer. Using the same procedure, this cytometer is capable of performing a number of other assays including: apoptosis, RFP and cell viability analysis using non-transduced cells.

Fluorometric assays of cell viability, gene expression and apoptosis have become key methodologies in biomedical research ${ }^{7-12}$. In the past, separate instrumentation has been used to obtain quantitative and visual information about cells. While quantitative information about cells within a heterogeneous population has been garnered through the use of flow cytometry, visual or qualitative information has been gathered through the use of fluorescence microscopy ${ }^{1-4}$. Here, we present a technology that bridges the gap between population and individual cell studies. Using the Tali Image-Based Cytometer, quantitative data and visual cell data about individual cells or cell populations can be collected simultaneously. The Tali instrument can be used for a range of applications including assessment of gene expression, apoptosis assays, studies of drug efficacy, and assessment of disease progression.

The Tali Image-Based Cytometer captures and analyzes bright-field, red fluorescence and green fluorescence images, allowing information about subpopulations of cells to be obtained. For example, in this assay, we were able to distinguish dead cells from live cells within the GFPexpressing cell population using Dead Cell Red dye. This gives accuracy in the measurement of viable cells expressing GFP, and identifies the population of cells useable for downstream processing. It is important to note that the dead cells in the population could arise from various sources, including normal cell death in culture or cell death caused by environmental conditions (e.g., trypsinization or death induced by the transfection/transduction method chosen).

Here we demonstrate the use of the Tali cytometer with U2OS cells. Similar methods can be performed using most mammalian and insect cells. For each cell line, the Tali cytometer produced quantitative GFP expression data, which is not possible with a visual inspection of the cells using a standard fluorescence microscope. Although these results are comparable to flow cytometry, they are collected in a fraction of the time. The cytometer is capable of accurately counting cells between $5 \mu \mathrm{m}$ to $60 \mu \mathrm{m}$ in diameter, ideally at a concentration of $1 \times 10^{5}$ to $1 \times 10^{7}$ cells/mL.

It's important to point out that most standard staining protocols, including the Dead Cell Red staining protocol described here, use propidium iodide to distinguish live and dead cells. This presents a challenge when assessing viability in cells that have been permeabilized for staining of intracellular markers, since PI will stain any cell with a compromised membrane. Since any fluorescent dye or protein that can be detected with the green or red fluorescence channels can be analyzed on the Tali Image-Based Cytometer, future studies may explore the use of alternate dyes, such as amine reactive viability dyes ${ }^{4}$. A 2006 study by Perfetto et al. ${ }^{12}$ found these dyes to be easy to use and reproducible in discriminating live and dead cell populations.

Note that the Tali has limitations with respect to the types of assays it can perform. For example, since the objective used by the instrument is 4X, counting operations are limited to larger cell types: sub-cellular structures such as mitochondria or vesicles, and bacterial cells cannot be quantified. Also note that the detection channels in Tali are limited to fluorophores that will excite and emit within the channels. While YFP and bright mCherry signals can be detected, dimmer mCherry signals cannot. Finally, the Tali only analyzes cells in suspension. It will not accurately count cells adhering to a slide. Moreover, while there is only limited capability for counting cells that are irregularly shaped, including some yeast and primary cells.

In addition, future studies will likely address the use of this system in assessing the expression of intracellular markers. Individual dyes can be checked for spectral overlap with adjacent channels, using Invitrogen's online SpectraViewer tool (www.invitrogen.com/spectraviewer). Given the newness of this technology, the full range of applications has yet to be discovered.

In summary, the Tali Image-Based Cytometer demonstrated here presents a new technology that allows for simultaneous visualization and analysis of cell populations, enabling assessment of individual cells as well as cell populations in a compact format with a simple workflow.

\section{Disclosures}

Krissy Remple and Laurel Stone are employees of Life Technologies who produced the instrument used in this article. Life Technologies has sponsored the production of this video-article.

\section{References}

1. Godfrey, W.L., Hill, D.M., Kilgore, J.A., Buller, G.M., Bradford, J.A., Gray, D.R., Clements, I., Oakleaf, K., Salisbury, J.J., Ignatius, M.J., \& Janes, M.S. Complementarity of Flow Cytometry and Fluorescence Microscopy. Microsc. Microanal. 11, $246-247$ (2005).

2. Mittag, A., Pinto, F.E., Endringer, D.C., Tarnok, A., \& Lenz, D. Cellular analysis by open-source software for affordable cytometry. Scanning. 33, 33-40 (2011).

3. Patterson, G.H., Knobel, S.M., Sharif, W.D., Kain, S.R. \& Piston, D.W. Use of the green fluorescent protein and its mutants in quantitative fluorescence microscopy. Biophys. J. 73, 2782-90 (1997).

4. Smyth, P.G. \& Berman, S.A. Markers of apoptosis: methods for elucidating the mechanism of apoptotic cell death from the nervous system. Biotechniques. 32, 648-50, 652, 654 passim (2002).

5. Vermes, I., Haanen, C., Steffens-Nakken, H., \& Reutelingsperger, C. A novel assay for apoptosis. Flow cytometric detection of phosphatidylserine expression on early apoptotic cells using fluorescein labelled Annexin V. J. Immunol. Methods. 184, 39-51 (1995).

6. Koopman, G., et al. Annexin V for flow cytometric detection of phosphatidylserine expression on B cells undergoing apoptosis. Blood. 84, 1415-20 (1994). 
7. Tanke, H.J., van der Linden, P.W., \& Langerak, J. Alternative fluorochromes to ethidium bromide for automated read out of cytotoxicity tests. J. Immunol. Methods. 52, 91-6 (1982).

8. King, M.A. Detection of dead cells and measurement of cell killing by flow cytometry. J. Immunol. Methods. 243, 155-66 (2000).

9. Jones, K.H. \& Kniss, D.A. Propidium iodide as a nuclear counterstain for immunofluorescence studies on cells in culture. J. Histochem. Cytochem. 35, 123-5 (1987).

10. Dell'Arciprete, R., et al. High-efficiency expression gene cloning by flow cytometry. J. Histochem. Cytochem. 44, 629-40 (1996).

11. Lamm, G.M., Steinlein, P., Cotten, M., \& Christofori, G. A rapid, quantitative and inexpensive method for detecting apoptosis by flow cytometry in transiently transfected cells. Nucleic. Acids. Res. 25, 4855-7 (1997).

12. Perfetto, S.P., et al. Amine reactive dyes: an effective tool to discriminate live and dead cells in polychromatic flow cytometry. J. Immunol. Methods. 313, 199-208 (2006). 